\title{
Optimal Monetary Policy Under Uncertainty: A Markov Jump-Linear-Quadratic Approach
}

\author{
Lars E.O. Svensson and Noah Williams
}

\begin{abstract}
This paper studies the design of optimal monetary policy under uncertainty using a Markov jumplinear-quadratic (MJLQ) approach. To approximate the uncertainty that policymakers face, the authors use different discrete modes in a Markov chain and take mode-dependent linear-quadratic approximations of the underlying model. This allows the authors to apply a powerful methodology with convenient solution algorithms that they have developed. They apply their methods to analyze the effects of uncertainty and potential gains from experimentation for two sources of uncertainty in the New Keynesian Phillips curve. The examples highlight that learning may have sizable effects on losses and, although it is generally beneficial, it need not always be so. The experimentation component typically has little effect and in some cases it can lead to attenuation of policy. (JEL E42, E52, E58)
\end{abstract}

Federal Reserve Bank of St. Louis Review, July/August 2008, 90(4), pp. 275-93.

I have long been interested in the analysis of monetary policy under uncertainty. The problems arise from what we do not know; we must deal with the uncertainty from the base of what we do know...

The Fed faces many uncertainties, and must adjust its one policy instrument to navigate as best it can this sea of uncertainty. Our fundamental principle is that we must use that one policy instrument to achieve long-run price stability...

My bottom line is that market participants should concentrate on the fundamentals. If the bond traders can get it right, they'll do most of the stabilization work for us, and we at the Fed can sit back and enjoy life.

$$
\begin{array}{r}
- \text {-William Poole (1998), } \\
\text { President of the Federal Reserve Bank of St. Louis }
\end{array}
$$

(1998-2008)

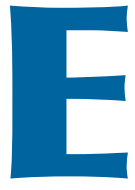

arly in his tenure as president of the Federal Reserve Bank of St. Louis, William Poole laid out some of the issues that policymakers face when deciding on policy, as reflected in the quotations here. In this paper we take up some of these issues, applying a framework to help policymakers navigate the "sea of uncertainty." We focus particularly on the issue of the knowledge and beliefs of the policymakers and the private sector-showing how both groups of agents learn from their observations and how this may or may not lead to enhanced economic stability. We also address the extent to which policymakers should "sit back" or, instead, actively intervene in markets in order to gain knowledge to help mitigate future uncertainty.

In previous work, Svensson and Williams (2007a,b), we have developed methods to study optimal policy in Markov jump-linear-quadratic

\footnotetext{
Lars E.O. Svensson is deputy governor of the Sveriges Riksbank and a professor of economics at Princeton University. Noah Williams is an assistant professor of economics at Princeton University. The authors thank James Bullard, Timothy Cogley, Andrew Levin, and William Poole for comments on this paper. Financial support from the National Science Foundation is gratefully acknowledged.

(C) 2008, The Federal Reserve Bank of St. Louis. The views expressed in this article are those of the author(s) and do not necessarily reflect the views of the Federal Reserve System, the Board of Governors, or the regional Federal Reserve Banks, or the Executive Board of Sveriges Riksbank. Articles may be reprinted, reproduced, published, distributed, displayed, and transmitted in their entirety if copyright notice, author name(s), and full citation are included. Abstracts, synopses, and other derivative works may be made only with prior written permission of the Federal Reserve Bank of St. Louis.
} 
(MJLQ) models with forward-looking variables: models with conditionally linear dynamics and conditionally quadratic preferences, where the matrices in both preferences and dynamics are random. In particular, each model has multiple modes, a finite collection of different possible values for the matrices, whose evolution is governed by a finite-state Markov chain. In our previous work, we have discussed how these modes could be structured to capture many different types of uncertainty relevant for policymakers. Here we put those suggestions into practice in a simple benchmark policy model.

In a first paper, Svensson and Williams (2007a), we studied optimal policy design in MJLQ models when policymakers can or cannot observe the current mode, but we abstracted from any learning and inference about the current mode. Although in many cases the optimal policy under no learning (NL) is not a normatively desirable policy, it serves as a useful benchmark for our later policy analyses. In a second paper, Svensson and Williams (2007b), we focused on learning and inference in the more relevant situation, particularly for the model-uncertainty applications which interest us, in which the modes are not directly observable. Thus, decisionmakers must filter their observations to make inferences about the current mode. As in most Bayesian learning problems, the optimal policy thus typically includes an experimentation component reflecting the endogeneity of information. This class of problems has a long history in economics, and it is well-known that solutions are difficult to obtain. We developed algorithms to solve numerically for the optimal policy. ${ }^{1}$ Due to the

\footnotetext{
1 In addition to the classic literature (on such problems as a monopolist learning its demand curve), Wieland (2000 and 2006) and Beck and Wieland (2002) have recently examined Bayesian optimal policy and optimal experimentation in a context similar to ours but without forward-looking variables. Tesfaselassie, Schaling, and Eijffinger (2006) examine passive and active learning in a simple model with a forward-looking element in the form of a long interest rate in the aggregate-demand equation. Ellison and Valla (2001) and Cogley, Colacito, and Sargent (2007) study situations like ours but where the expectational component is as in the Lucas-supply curve ( $\mathrm{E}_{t-1} \pi_{t}$, for example) rather than our forwardlooking case $\left(\mathrm{E}_{t} \pi_{t+1}\right.$, for example). More closely related to our present paper, Ellison (2006) analyzes active and passive learning in a New Keynesian model with uncertainty about the slope of the Phillips curve.
}

curse of dimensionality, the Bayesian optimal policy (BOP) is feasible only in relatively small models. Confronted with these difficulties, we also considered adaptive optimal policy (AOP). ${ }^{2}$ In this case, in each period the policymaker does update the probability distribution of the current mode in a Bayesian way, but the optimal policy is computed each period under the assumption that the policymaker will not learn in the future from observations. In our setting, the AOP is significantly easier to compute, and in many cases provides a good approximation to the BOP. Moreover, the AOP analysis is of some interest in its own right because it is closely related to specifications of adaptive learning that have been widely studied in macroeconomics (see Evans and Honkapohja, 2001, for an overview). Further, the AOP specification rules out the experimentation that some may view as objectionable in a policy context. ${ }^{3}$

In this paper, we apply our methodology to study optimal monetary policy design under uncertainty in dynamic stochastic general equilibrium (DSGE) models. We begin by summarizing the main findings from our previous work, leading to implementable algorithms for analyzing policy in MJLQ models. We then turn to examples that highlight the effects of learning and experimentation for two sources of uncertainty in the benchmark New Keynesian Phillips curve. In this model we compare and contrast optimal policies under NL, AOP, and BOP. We analyze whether learning is beneficial-it is not always so, a fact which at least partially reflects our assumption of symmetric information between the policymakers and the public - and then quantify the additional gains from experimentation. ${ }^{4}$ We find

\footnotetext{
2 What we call optimal policy under no learning, adaptive optimal policy, and Bayesian optimal policy have in the literature also been referred to as myopia, passive learning, and active learning, respectively.

${ }^{3}$ In addition, AOP is useful for technical reasons because it gives us a good starting point for our more intensive numerical calculations in the BOP case.

4 In addition to our own previous work, MJLQ models have been widely studied in the control-theory literature for the special case when the model modes are observable and there are no forwardlooking variables (see Costa, Fragoso, and Marques, 2005, and the references therein); do Val and Başar (1999) provide an application of an adaptive-control MJLQ problem in economics. More recently, Zampolli (2006) has used such an MJLQ model to examine mone-
} 
that the experimentation component is typically small. Recognizing the informational component of policy actions often leads policy to be slightly more aggressive, but, somewhat surprisingly, in one example here it leads to a less aggressive optimal policy.

The paper is organized as follows: The next section presents the MJLQ framework and summarizes our earlier work. The third section presents our analysis of learning and experimentation in a simple benchmark New Keynesian model. The fourth section presents some conclusions and suggestions for further work.

\section{MJLQ ANALYSIS OF OPTIMAL POLICY}

This section summarizes our earlier work, Svensson and Williams (2007a,b).

\section{An MJLQ Model}

We consider an MJLQ model of an economy with forward-looking variables. The economy has a private sector and a policymaker. We let $X_{t}$ denote an $n_{x}$ vector of predetermined variables in period $t, x_{t}$ an $n_{x}$ vector of forward-looking variables, and $i_{t}$ an $n_{x}$ vector of (policymaker) instruments (control variables). ${ }^{5}$ We let model uncertainty be represented by $n_{j}$ possible modes and let $j_{t} \in N_{j} \equiv\left\{1,2, \ldots, n_{j}\right\}$ denote the mode in

tary policy under shifts between regimes with and without an asset-market bubble. Blake and Zampolli (2006) provide an extension of the MJLQ model with observable modes to include forwardlooking variables and present an algorithm for the solution of an equilibrium resulting from optimization under discretion. Svensson and Williams (2007a) provide a more general extension of the MJLQ framework with forward-looking variables and present algorithms for the solution of an equilibrium resulting from optimization under commitment in a timeless perspective as well as arbitrary time-varying or time-invariant policy rules, using the recursive saddlepoint method of Marcet and Marimon (1998). They also provide two concrete examples: an estimated backward-looking model (a three-mode variant of Rudebusch and Svensson, 1999) and an estimated forward-looking model (a three-mode variant of Lindé, 2005). Svensson and Williams (2007a) also extend the MJLQ framework to the more realistic case of unobservable modes, although without introducing learning and inference about the probability distribution of modes. Svensson and Williams (2007b) focus on learning and experimentation in the MJLQ framework.

5 The first component of $X_{t}$ may be unity, in order to allow for mode-dependent intercepts in the model equations. period $t$. The model of the economy can then be written

(1) $X_{t+1}=A_{11 j_{t+1}} X_{t}+A_{12 j_{t+1}} x_{t}+B_{1 j_{t+1}} i_{t}+C_{1 j_{t+1}} \varepsilon_{t+1}$,

(2) $\mathrm{E}_{t} H_{j_{t+1}} x_{t+1}=A_{21 j_{t}} X_{t}+A_{22 j_{t}} x_{t}+B_{2 j_{t}} i_{t}+C_{2 j_{t}} \varepsilon_{t}$,

where $\varepsilon_{t}$ is a multivariate normally distributed random i.i.d. $n_{\varepsilon}$ vector of shocks with mean zero and contemporaneous covariance matrix $I_{n_{\varepsilon}}$. The matrices $A_{11 j}, A_{12 j}, \ldots, C_{2 j}$ have the appropriate dimensions and depend on the mode $j$. Because a structural model here is simply a collection of matrices, each mode can represent a different model of the economy. Thus, uncertainty about the prevailing mode is model uncertainty. ${ }^{6}$

Note that the matrices on the right side of (1) depend on the mode $j_{t+1}$ in period $t+1$, whereas the matrices on the right side of (2) depend on the mode $j_{t}$ in period $t$. Equation (1) then determines the predetermined variables in period $t+1$ as a function of the mode and shocks in period $t+1$ and the predetermined variables, forward-looking variables, and instruments in period $t$. Equation (2) determines the forward-looking variables in period $t$ as a function of the mode and shocks in period $t$, the expectations in period $t$ of next period's mode and forward-looking variables, and the predetermined variables and instruments in period $t$. The matrix $A_{22 j}$ is nonsingular for each $j \in N_{j}$.

The mode $j_{t}$ follows a Markov process with the transition matrix

$$
P \equiv\left\lfloor P_{j k}\right\rfloor \cdot 7
$$

The shocks $\varepsilon_{t}$ are mean zero and i.i.d. with probability density $\varphi$; and without loss of generality we assume that $\varepsilon_{t}$ is independent of $j_{t}{ }^{8}$ We also assume that $C_{1 j} \varepsilon_{t}$ and $c_{2 k} \varepsilon_{t}$ are independent for

6 See also Svensson and Williams (2007a), where we show how many different types of uncertainty can be mapped into our MJLQ framework

7 Obvious special cases are $P=I_{n_{j}}$, when the modes are completely persistent, and $P_{j}=\bar{p}^{\prime}\left(j \in N_{j}\right)$, when the modes are serially i.i.d. with probability distribution $\bar{p}$.

8 Because mode-dependent intercepts (as well as mode-dependent standard deviations) are allowed in the model, we can still incorporate additive mode-dependent shocks. 
all $j, k \in N_{j}$. These shocks, along with the modes, are the driving forces in the model. They are not directly observed. For technical reasons, it is convenient but not necessary that they are independent. We let $p_{t}=\left(p_{1 t}, \ldots, p_{n_{i} t}\right)^{\prime}$ denote the true probability distribution of $j_{t}$ in period $t$. We let $p_{t+\tau \mid t}$ denote the policymaker and private sector estimate in the beginning of period $t$ of the probability distribution in period $t+\tau$. The prediction equation for the probability distribution is

$$
p_{t+1 \mid t}=P^{\prime} p_{t \mid t} .
$$

We let the operator $E_{t}[\cdot]$ in the expression $E_{t} H_{j_{t+1}} X_{t+1}$ on the left side of (2) denote expectations in period $t$ conditional on policymaker and private sector information in the beginning of period $t$, including $X_{t}, i_{t}$, and $p_{t \mid t}$ but excluding $j_{t}$ and $\varepsilon_{t}$. Thus, the maintained assumption is symmetric information between the policymaker and the (aggregate) private sector. Because forwardlooking variables will be allowed to depend on $j_{t}$, parts of the private sector, but not the aggregate private sector, may be able to observe $j_{t}$ and parts of $\varepsilon_{t}$. Note that although we focus on the determination of the optimal policy instrument, $i_{t}$, our results also show how private sector choices as embodied in $x_{t}$ are affected by uncertainty and learning. The precise informational assumptions and the determination of $p_{t \mid t}$ will be specified below.

We let the policymaker intertemporal loss function in period $t$ be

$$
\mathrm{E}_{t} \sum_{\tau=0}^{\infty} \delta^{\tau} L\left(X_{t+\tau}, X_{t+\tau}, i_{t+\tau}, j_{t+\tau}\right),
$$

where $\delta$ is a discount factor satisfying $0<\delta<1$, and the period loss, $L\left(X_{t}, X_{t}, i_{t}, j_{t}\right)$, satisfies

$$
L\left(X_{t}, x_{t}, i_{t}, j_{t}\right) \equiv\left[\begin{array}{c}
X_{t} \\
x_{t} \\
i_{t}
\end{array}\right]^{\prime} W_{j_{t}}\left[\begin{array}{c}
X_{t} \\
x_{t} \\
i_{t}
\end{array}\right],
$$

where the matrix $W_{j}\left(j \in N_{j}\right)$ is positive semidefinite. We assume that the policymaker optimizes under commitment in a timeless perspective. As explained below, we will then add the term

$$
\Xi_{t-1} \frac{1}{\delta} \mathrm{E}_{t} H_{j_{t}} x_{t}
$$

to the intertemporal loss function in period $t$. As we shall see below, the $n_{x}$ vector $\Xi_{t-1}$ is the vector of Lagrange multipliers for equation (2) from the optimization problem in period $t-1$. For the special case when there are no forward-looking variables $\left(n_{x}=0\right)$, the model consists of (1) only, without the term $A_{12 j_{t+1}} X_{t}$; the period loss function depends on $X_{t}, i_{t}$, and $j_{t}$ only; and there is no role for the Lagrange multipliers, $\Xi_{t-1}$, or the term (6).

\section{Approximate MJLQ models}

Although in this paper we start with an MJLQ model, it is natural to ask where such a model comes from, as usual formulations of economic models are not of this type. However, the same type of approximation methods that are widely used to convert nonlinear models into their linear counterparts can also convert nonlinear models into MJLQ models. We analyze this issue in Svensson and Williams (2007a) and present an illustration as well. Here we briefly discuss the main ideas. Rather than analyze local deviations from a single steady state as in conventional linearizations, for an MJLQ approximation we analyze the local deviations from (potentially) separate, mode-dependent steady states. Standard linearizations are justified as asymptotically valid for small shocks, as an increasing time is spent in the vicinity of the steady state. Our MJLQ approximations are asymptotically valid for small shocks and persistent modes, as an increasing time is spent in the vicinity of each mode-dependent steady state. Thus, for slowly varying Markov chains, our MJLQ model provides accurate approximations of nonlinear models with Markov switching.

\section{Types of Optimal Policies}

We will distinguish three cases: (i) optimal policy when there is no learning (NL), (ii) adaptive optimal policy (AOP), and (iii) Bayesian optimal policy (BOP). By NL, we refer to a situation when the policymaker and the aggregate private sector have a probability distribution $p_{t \mid t}$ over the modes in period $t$ and update the probability 
distribution in future periods using the transition matrix only, so the updating equation is

$$
p_{t+1 \mid t+1}=P^{\prime} p_{t \mid t} \text {. }
$$

That is, the policymaker and the private sector do not use observations of the variables in the economy to update the probability distribution. The policymaker then determines optimal policy in period $t$ conditional on $p_{t \mid t}$ and (7). This is a variant of a case examined in Svensson and Williams (2007a).

By AOP, we refer to a situation when the policymaker in period $t$ determines optimal policy as in the NL case, but then uses observations of the realization of the variables in the economy to update the probability distribution according to Bayes's theorem. In this case, the instruments will generally have an effect on the updating of future probability distributions and through this channel separately affect the intertemporal loss. However, the policymaker does not exploit this channel in determining optimal policy. That is, the policymaker does not do any conscious experimentation. By BOP, we refer to a situation when the policymaker acknowledges that the current instruments will affect future inference and updating of the probability distribution and calculates optimal policy taking this separate channel into account. Therefore, BOP includes optimal experimentation, where for instance the policymaker may pursue policy that increases losses in the short run but improves the inference of the probability distribution and therefore lowers losses in the longer run.

\section{Optimal Policy with No Learning}

We first consider the NL case. Svensson and Williams (2007a) derive the equilibrium under commitment in a timeless perspective for the case when $X_{t}, x_{t}$, and $i_{t}$ are observable in period $t, j_{t}$ is unobservable, and the updating equation for $p_{t \mid t}$ is given by (7). Observations of $X_{t}, X_{t}$, and $i_{t}$ are then not used to update $p_{t \mid t}$.

It will be useful to replace equation (2) with the two equivalent equations,

$$
\mathrm{E}_{t} H_{j_{t+1}} x_{t+1}=z_{t}
$$

(9)

$$
0=A_{21 j_{t}} X_{t}+A_{22 j_{t}} x_{t}-z_{t}+B_{2 j_{t}} i_{t}+C_{2 j_{t}} \varepsilon_{t},
$$

where we introduce the $n_{x}$ vector of additional forward-looking variables, $z_{t}$. Introducing this vector is a practical way of keeping track of the expectations term on the left side of (2). Furthermore, it will be practical to use (9) and solve $x_{t}$ as a function of $X_{t}, z_{t}, i_{t}, j_{t}$, and $\varepsilon_{t}$ :

$$
\begin{aligned}
x_{t} & =\tilde{X}\left(X_{t}, z_{t}, i_{t}, j_{t}, \varepsilon_{t}\right) \\
& \equiv A_{22 j_{t}}^{-1}\left(z_{t}-A_{21 j_{t}} X_{t}-B_{2 j_{t}} i_{t}-C_{2 j_{t}} \varepsilon_{t}\right) .
\end{aligned}
$$

We note that, for given $j_{t}$, this function is linear in $X_{t}, z_{t}, i_{t}$, and $\varepsilon_{t}$.

In order to solve for the optimal decisions, we use the recursive saddlepoint method (see Marcet and Marimon, 1998, Svensson and Williams, 2007a, and Svensson, 2007, for details of the recursive saddlepoint method). Thus, we introduce Lagrange multipliers for each forward-looking equation, the lagged values of which become state variables and reflect costs of commitment, while the current values become control variables. The dual period loss function can be written

$$
\begin{aligned}
& \mathrm{E}_{t} \tilde{L}\left(\tilde{X}_{t}, z_{t}, i_{t}, \gamma_{t}, j_{t}, \varepsilon_{t}\right) \\
& \equiv \sum_{j} p_{j t t t} \int \tilde{L}\left(\tilde{X}_{t}, z_{t}, i_{t}, \gamma_{t}, j, \varepsilon_{t}\right) \varphi\left(\varepsilon_{t}\right) d \varepsilon_{t},
\end{aligned}
$$

where $\tilde{X}_{t} \equiv\left(X_{t}^{\prime}, \Xi_{t-1}^{\prime}\right)^{\prime}$ is the $\left(n_{X}+n_{x}\right)$ vector of extended predetermined variables (that is, including the $n_{x}$ vector $\left.\Xi_{t-1}\right), \gamma_{t}$ is an $n_{x}$ vector of Lagrange multipliers, $\varphi(\cdot)$ denotes a generic probability density function (for $\varepsilon_{t}$, the standard normal density function), and

$$
\begin{aligned}
& \tilde{L}\left(\tilde{X}_{t}, z_{t}, i_{t}, \gamma_{t}, j_{t}, \varepsilon_{t}\right) \equiv L\left[X_{t}, \tilde{x}\left(X_{t}, z_{t}, i_{t}, j_{t}, \varepsilon_{t}\right), i_{t}, j_{t}\right] \\
& -\gamma_{t}^{\prime} z_{t}+\Xi_{t-1}^{\prime} \frac{1}{\delta} H_{j_{t}} \tilde{X}\left(X_{t}, z_{t}, i_{t}, j_{t}, \varepsilon_{t}\right) .
\end{aligned}
$$

As discussed in Svensson and Williams (2007a), the failure of the law of iterated expectations leads us to introduce the collection of value functions, $\hat{V}\left(s_{t}, j\right)$, that condition on the mode, whereas the value function $\tilde{V}\left(s_{t}\right)$ averages over these and represents the solution of the dual optimization problem. The somewhat unusual 
Bellman equation for the dual problem can be written

$$
\begin{aligned}
& \tilde{V}\left(s_{t}\right) \equiv \mathrm{E}_{t} \hat{V}\left(s_{t}, j_{t}\right) \equiv \sum_{j} p_{j t t t} \hat{V}\left(s_{t}, j\right) \\
& \left.=\max _{\gamma_{t}} \min _{\left(z_{t}, i_{t}\right)} \mathrm{E}_{t}\left\{\begin{array}{l}
\tilde{L}\left(\tilde{X}_{t}, z_{t}, i_{t}, \gamma_{t}, j_{t}, \varepsilon_{t}\right) \\
+\delta \hat{V}\left[g\left(\begin{array}{l}
s_{t}, z_{t}, i_{t}, \gamma_{t}, j_{t}, \\
\varepsilon_{t}, j_{t+1}, \varepsilon_{t+1}
\end{array}\right), j_{t+1}\right.
\end{array}\right]\right\} \\
& \equiv \max _{\gamma_{t}} \min _{\left(z_{t}, i_{t}\right)} \sum_{j} p_{j t t t}\left[\begin{array}{l}
\tilde{L}\left(\tilde{X}_{t}, z_{t}, i_{t}, \gamma_{t}, j, \varepsilon_{t}\right) \\
+\delta \sum_{k} P_{j k} \hat{V}\left[g\left(\begin{array}{l}
s_{t}, z_{t}, i_{t}, \gamma_{t}, \\
j, \varepsilon_{t}, k, \varepsilon_{t+1}
\end{array}\right), k\right]
\end{array}\right] \\
& \varphi\left(\varepsilon_{t}\right) \varphi\left(\varepsilon_{t+1}\right) d \varepsilon_{t} d \varepsilon_{t+1},
\end{aligned}
$$

where $s_{t} \equiv\left(\tilde{X}_{t}^{\prime}, p_{t \mid t}^{\prime}\right)^{\prime}$ denotes the perceived state of the economy (it includes the perceived probability distribution, $p_{t \mid t}$, but not the true mode) and $\left(s_{t}, j_{t}\right)$ denotes the true state of the economy (it includes the true mode of the economy). As we discuss in more detail below, it is necessary to include the mode $j_{t}$ in the state vector because the beliefs do not satisfy the law of iterated expectations. In the BOP case, beliefs do satisfy this property, so the state vector is simply $s_{t}$. Also note that, in the Bellman equation, we require that all the choice variables respect the information constraints and thus depend on the perceived state, $s_{t}$, but not the mode $j_{t}$ directly.

The optimization is subject to the transition equation for $X_{t}$,

$$
\begin{aligned}
& X_{t+1}=A_{11 j_{t+1}} X_{t}+A_{12 j_{t+1}} \tilde{X}\left(X_{t}, z_{t}, i_{t}, j_{t}, \varepsilon_{t}\right) \\
& +B_{1 j_{t+1}} i_{t}+C_{1 j_{t+1}} \varepsilon_{t+1},
\end{aligned}
$$

where we have substituted $\tilde{x}\left(X_{t}, z_{t}, i_{t}, j_{t}, \varepsilon_{t}\right)$ for $x_{t}$; the new dual transition equation for $\Xi_{t}$,

$$
\Xi_{t}=\gamma_{t}
$$

and the transition equation (7) for $p_{t \mid t}$. Combining equations, we have the transition for $s_{t}$ :

$$
\begin{aligned}
s_{t+1} & \equiv\left[\begin{array}{c}
X_{t+1} \\
\Xi_{t} \\
p_{t+1 \mid t+1}
\end{array}\right]=g\left(s_{t}, z_{t}, i_{t}, \gamma_{t}, j_{t}, \varepsilon_{t}, j_{t+1}, \varepsilon_{t+1}\right) \\
& {\left[\begin{array}{c}
A_{11 j_{t+1}} X_{t}+A_{12 j_{t+1}} \tilde{x}\left(X_{t}, z_{t}, i_{t}, j, \varepsilon_{t}\right) \\
+B_{1 j_{t+1}} i_{t}+C_{1 j_{t+1}} \varepsilon_{t+1} \\
\gamma_{t} \\
P^{\prime} p_{t \mid t}
\end{array}\right] . }
\end{aligned}
$$

It is straightforward to see that the solution of the dual optimization problem (12) is linear in $\tilde{X}_{t}$ for given $p_{t \mid t}, j_{t}$ :

$$
\begin{aligned}
& {\left[\begin{array}{c}
z_{t} \\
i_{t} \\
\gamma_{t}
\end{array}\right]=\left[\begin{array}{c}
z\left(s_{t}\right) \\
i\left(s_{t}\right) \\
\gamma\left(s_{t}\right)
\end{array}\right]=F\left(p_{t \mid t}\right) \tilde{X}_{t} \equiv\left[\begin{array}{l}
F_{z}\left(p_{t \mid t}\right) \\
F_{i}\left(p_{t \mid t}\right) \\
F_{\gamma}\left(p_{t \mid t}\right)
\end{array}\right] \tilde{X}_{t},} \\
& x_{t}=x\left(s_{t}, j_{t}, \varepsilon_{t}\right) \equiv \tilde{X}\left(X_{t}, z\left(s_{t}\right), i\left(s_{t}\right), j_{t}, \varepsilon_{t}\right) \\
& \equiv F_{x \tilde{X}}\left(p_{t \mid t}, j_{t}\right) \tilde{X}_{t}+F_{x \varepsilon}\left(p_{t \mid t}, j_{t}\right) \varepsilon_{t} .
\end{aligned}
$$

This solution is also the solution to the original primal optimization problem. We note that $x_{t}$ is linear in $\varepsilon_{t}$ for given $p_{t \mid t}$ and $j_{t}$. The equilibrium transition equation is then given by

$$
\begin{aligned}
s_{t+1} & =\hat{g}\left(s_{t}, j_{t}, \varepsilon_{t}, j_{t+1}, \varepsilon_{t+1}\right) \\
& \equiv g\left[s_{t}, z\left(s_{t}\right), i\left(s_{t}\right), \gamma\left(s_{t}\right), j_{t}, \varepsilon_{t}, j_{t+1}, \varepsilon_{t+1}\right] .
\end{aligned}
$$

As can be easily verified, the (unconditional) dual value function $\tilde{V}\left(s_{t}\right)$ is quadratic in $\tilde{X}_{t}$ for given $p_{t \mid t}$, taking the form

$$
\tilde{V}\left(s_{t}\right) \equiv \tilde{X}_{t}^{\prime} \tilde{V}_{\tilde{X} \tilde{X}}\left(p_{t \mid t}\right) \tilde{X}_{t}+w\left(p_{t \mid t}\right) .
$$

The conditional dual value function, $\hat{V}\left(s_{t}, j_{t}\right)$, gives the dual intertemporal loss conditional on the true state of the economy, $\left(s_{t}, j_{t}\right)$. It follows that this function satisfies

$$
\begin{aligned}
& \hat{V}\left(s_{t}, j\right) \equiv \int\left[\begin{array}{l}
\tilde{L}\left(\tilde{X}_{t}, z\left(s_{t}\right), i\left(s_{t}\right), \gamma\left(s_{t}\right), j, \varepsilon_{t}\right) \\
+\delta \sum_{k} P_{j k} \hat{V}\left[\hat{g}\left(s_{t}, j, \varepsilon_{t}, k, \varepsilon_{t+1}\right), k\right]
\end{array}\right] \\
& \varphi\left(\varepsilon_{t}\right) \varphi\left(\varepsilon_{t+1}\right) d \varepsilon_{t} d \varepsilon_{t+1} \quad\left(j \in N_{j}\right) .
\end{aligned}
$$


The function $\hat{V}\left(s_{t}, j_{t}\right)$ is also quadratic in $\tilde{X}_{t}$ for given $p_{t \mid t}$ and $j_{t}$ :

$$
\hat{V}\left(s_{t}, j_{t}\right) \equiv \tilde{X}_{t}^{\prime} \hat{V}_{\tilde{X} \tilde{X}}\left(p_{t \mid t}, j_{t}\right) \tilde{X}_{t}+\hat{W}\left(p_{t \mid t}, j_{t}\right) .
$$

It follows that we have

$$
\begin{gathered}
\tilde{V}_{\tilde{X} \tilde{X}}\left(p_{t \mid t}\right) \equiv \sum_{j} p_{j t t t} \hat{V}_{\tilde{X} \tilde{X}}\left(p_{t \mid t}, j\right), \\
w\left(p_{t \mid t}\right) \equiv \sum_{j} p_{j t t \mid} \hat{W}\left(p_{t \mid t}, j\right) .
\end{gathered}
$$

Although we find the optimal policies from the dual problem, in order to measure true expected losses, we are interested in the value function for the primal problem (with the original, unmodified loss function). This value function, with the period loss function $E_{t} L\left(X_{t}, X_{t}, i_{t}, j_{t}\right)$, rather than $E_{t} \tilde{L}\left(\tilde{X}_{t}, z_{t}, i_{t}, \gamma_{t}, j_{t}, \varepsilon_{t}\right)$, satisfies

$$
\begin{aligned}
V\left(s_{t}\right) & \equiv \tilde{V}\left(s_{t}\right)-\Xi_{t-1}^{\prime} \frac{1}{\delta} \sum_{j} p_{j t t t} H_{j} \int x\left(s_{t}, j, \varepsilon_{t}\right) \varphi\left(\varepsilon_{t}\right) d \varepsilon_{t} \\
& =\tilde{V}\left(s_{t}\right)-\Xi_{t-1}^{\prime} \frac{1}{\delta} \sum_{j} p_{j t t t} H_{j} x\left(s_{t}, j, 0\right),
\end{aligned}
$$

where the second equality follows because $x\left(s_{t}, j_{t}, \varepsilon_{t}\right)$ is linear in $\varepsilon_{t}$ for given $s_{t}$ and $j_{t}$. It is quadratic in $\tilde{X}_{t}$ for given $p_{t \mid t}$ :

$$
V\left(s_{t}\right) \equiv \tilde{X}_{t}^{\prime} V_{\tilde{X} \tilde{X}}\left(p_{t \mid t}\right) \tilde{X}_{t}+w\left(p_{t \mid t}\right)
$$

(the scalar $w\left(p_{t \mid t}\right)$ in the primal value function is obviously identical to that in the dual value function). This is the value function conditional on $\tilde{X}_{t}$ and $p_{t \mid t}$ after $X_{t}$ has been observed but before $x_{t}$ has been observed, taking into account that $j_{t}$ and $\varepsilon_{t}$ are not observed. Hence, the second term on the right side of (19) contains the expectation of $H_{j_{t}} X_{t}$ conditional on that information. ${ }^{9}$

Svensson and Williams (2007a,b) present algorithms to compute the solution and the primal and dual value functions for the NL case. For future reference, we note that the value function for the primal problem also satisfies

\footnotetext{
9 To be precise, the observation of $X_{t}$, which depends on $C_{1 j} \varepsilon_{t}$, allows some inference of $\varepsilon_{t}, \varepsilon_{t \mid t}, X_{t}$ will depend on $j_{t}$ and on $\varepsilon_{t}$, but on $\varepsilon_{t}$ only through $C_{2 j} \varepsilon_{t}$. By assumption $C_{1 j} \varepsilon_{t}$ and $C_{2 k} \varepsilon_{t}$ are independent. Hence, any observation of $X_{t}$ and $C_{1 j} \varepsilon_{t}$ does not convey any information about $C_{2 j} \varepsilon_{t}$, so $E_{t} C_{2 j} \varepsilon_{t}=0$.
}

$$
V\left(s_{t}\right) \equiv \sum_{j} p_{j t t t} \breve{V}\left(s_{t}, j\right),
$$

where the conditional value function, $\breve{V}\left(s_{t}, j_{t}\right)$, satisfies

$$
\begin{aligned}
& \breve{V}\left(s_{t}, j\right)=\int\left\{\begin{array}{l}
L\left[X_{t}, x\left(s_{t}, j, \varepsilon_{t}\right), i\left(s_{t}\right), j\right] \\
+\delta \sum_{k} P_{j k} \breve{V}\left[\hat{g}\left(s_{t}, j, \varepsilon_{t}, k, \varepsilon_{t+1}\right), k\right]
\end{array}\right\} \\
& \varphi\left(\varepsilon_{t}\right) \varphi\left(\varepsilon_{t+1}\right) d \varepsilon_{t} d \varepsilon_{t+1} \quad\left(j \in N_{j}\right) .
\end{aligned}
$$

\section{Adaptive Optimal Policy}

Consider now the case of AOP, where the policymaker uses the same policy function as in the NL case but each period updates the probabilities that this policy is conditioned on. This case is thus simple to implement recursively, as we have already discussed how to solve for the optimal decisions and below we show how to update probabilities. However, the ex ante evaluation of expected loss is more complex, as we show below. In particular, we assume that $C_{2 j_{t}} \neq 0$ and that both $\varepsilon_{t}$ and $j_{t}$ are unobservable. The estimate $p_{t \mid t}$ is the result of Bayesian updating, using all information available, but the optimal policy in period $t$ is computed under the perceived updating equation (7). That is, the fact that the policy choice will affect future $p_{t+\tau \mid t+\tau}$ and that future expected loss will change when $p_{t+\tau \mid t+\tau}$ changes is disregarded. Under the assumption that the expectations on the left side of (2) are conditional on (7), the variables $z_{t}, i_{t}, \gamma_{t}$, and $x_{t}$ in period $t$ are still determined by (16) and (17).

In order to determine the updating equation for $p_{t \mid t}$, we specify an explicit sequence of information revelation as follows, in no less than nine steps. The timing assumptions are necessary in order to spell out the appropriate conditioning for decisions and updating of beliefs.

(i) The policymaker and the private sector enters period $t$ with the prior $p_{t \mid t-1}$. They know $X_{t-1}, x_{t-1}=x\left(s_{t-1}, j_{t-1}, \varepsilon_{t-1}\right), z_{t-1}=z\left(s_{t-1}\right), i_{t-1}=i\left(s_{t-1}\right)$, and $\Xi_{t-1}=\gamma\left(s_{t-1}\right)$ from the previous period.

(ii) In the beginning of period $t$, the mode $j_{t}$ and the vector of shocks $\varepsilon_{t}$ are realized. Then the vector of predetermined variables $X_{t}$ is realized according to (1). 
(iii) The policymaker and the private sector observe $X_{t}$. They then know $\tilde{X}_{t} \equiv\left(X_{t}^{\prime}, \Xi_{t-1}^{\prime}\right)^{\prime}$. They do not observe $j_{t}$ or $\varepsilon_{t}$.

(iv) The policymaker and the private sector update the prior $p_{t \mid t-1}$ to the posterior $p_{t \mid t}$ according to Bayes's theorem and the updating equation

$$
\begin{aligned}
& p_{j t \mid t}=\frac{\varphi\left(X_{t} \mid j_{t}=j, X_{t-1}, X_{t-1}, i_{t-1}, p_{t \mid t-1}\right)}{\varphi\left(X_{t} \mid X_{t-1}, X_{t-1}, i_{t-1}, p_{t \mid t-1}\right)} p_{j t t-1} \\
& \left(j \in N_{j}\right)
\end{aligned}
$$

where again $\varphi(\cdot)$ denotes a generic density function. ${ }^{10}$ Then the policymaker and the private sector know $s_{t} \equiv\left(\tilde{X}_{t}^{\prime}, p_{t \mid t}^{\prime}\right)^{\prime}$.

(v) The policymaker solves the dual optimization problem, determines $i_{t}=i\left(s_{t}\right)$, and implements/announces the instrument setting, $i_{t}$.

(vi) The private sector (and policymaker) expectations,

$$
z_{t}=\mathrm{E}_{t} H_{j_{t+1}} x_{t+1} \equiv \mathrm{E}\left(H_{j_{t+1}} x_{t+1} \mid s_{t}\right),
$$

are formed. In equilibrium, these expectations will be determined by (16). In order to understand their determination better, we look at this in some detail.

These expectations are by assumption formed before $x_{t}$ is observed. The private sector and the policymaker know that $x_{t}$ will in equilibrium be determined in the next step according to (17).

Hence, they can form expectations of the soon-tobe determined $x_{t}$ conditional on $j_{t}=j,{ }^{11}$

$$
x_{j t \mid t}=x\left(s_{t}, j, 0\right) .
$$

\footnotetext{
${ }^{10}$ The policymaker and private sector can also estimate the shocks $\varepsilon_{t \mid t}$ as$$
\varepsilon_{t \mid t}=\sum_{j} p_{j t \mid t} \varepsilon_{j t \mid t}
$$

where

$$
\varepsilon_{j t \mid t} \equiv X_{t}-A_{11 j} X_{t-1}-A_{12 j} X_{t-1}-B_{1_{j}} i_{t-1} \quad\left(j \in N_{j}\right) .
$$

However, because of the assumed independence of $C_{1 j} \varepsilon_{t}$ and $C_{2 k} \varepsilon_{t}$, $j, k \in N_{j}$, we do not need to keep track of $\varepsilon_{j t \mid t}$.

${ }^{11}$ Note that 0 instead of $\varepsilon_{j t \mid t}$ enters above. This is because the inference $\varepsilon_{i t \mid t}$ above is inference about $C_{1 j} \varepsilon_{t}$, whereas $x_{t}$ depends on $\varepsilon_{t}$ through $C_{2 j} \varepsilon_{t}$. Because we assume that $C_{1 j} \varepsilon_{t}$ and $C_{2 j} \varepsilon_{t}$ are independent, there is no inference of $C_{2 j} \varepsilon_{t}$ from observing $X_{t}$. Hence, $E_{t} C_{2 j} \varepsilon_{t}=0$. Because of the linearity of $x_{t}$ in $\varepsilon_{t}$, the integration of $x_{t}$ over $\varepsilon_{t}$ results in $x\left(s_{t}, j_{t}, 0_{t}\right)$.
}

The private sector and the policymaker can also infer $\Xi_{t}$ from

$$
\Xi_{t}=\gamma\left(s_{t}\right)
$$

This allows the private sector and the policymaker to form the expectations

$$
\begin{aligned}
z_{t} & =z\left(s_{t}\right)=\mathrm{E}_{t}\left[H_{j_{t+1}} x_{t+1} \mid s_{t}\right] \\
& =\sum_{j, k} P_{j k} p_{j t t t} H_{k} x_{k, t+1 \mid j t},
\end{aligned}
$$

where

$$
\begin{array}{r}
x_{k, t+1 \mid j t}=\int x\left(\left[\begin{array}{c}
A_{11 k} X_{t}+A_{12 k} x\left(s_{t}, j, \varepsilon_{t}\right) \\
+B_{1 k} i\left(s_{t}\right) \\
\Xi_{t} \\
P^{\prime} p_{t \mid t}
\end{array}\right], k, \varepsilon_{t+1}\right) \\
\varphi\left(\varepsilon_{t}\right) \varphi\left(\varepsilon_{t+1}\right) d \varepsilon_{t} d \varepsilon_{t+1} \\
=x\left(\left[\begin{array}{c}
A_{11 k} X_{t}+A_{12 k} x\left(s_{t}, j, 0\right)+B_{1 k} i\left(s_{t}\right) \\
\Xi_{t} \\
P^{\prime} p_{t \mid t}
\end{array}\right], k, 0\right),
\end{array}
$$

where we have exploited the linearity of $x_{t}=$ $x\left(s_{t}, j_{t}, \varepsilon_{t}\right)$ and $x_{t+1}=x\left(s_{t+1}, j_{t+1}, \varepsilon_{t+1}\right)$ in $\varepsilon_{t}$ and $\varepsilon_{t+1}$. Note that $z_{t}$ is, under AOP, formed conditional on the belief that the probability distribution in period $t+1$ will be given by $p_{t+1 \mid t+1}=P^{\prime} p_{t \mid t}$, not by the true updating equation that we are about to specify.

(vii) After the expectations $z_{t}$ have been formed, $x_{t}$ is determined as a function of $X_{t}, z_{t}, i_{t}$, $j_{t}$, and $\varepsilon_{t}$ by (10).

(viii) The policymaker and the private sector then use the observed $x_{t}$ to update $p_{t \mid t}$ to the new posterior $p_{t \mid t}^{+}$according to Bayes's theorem, via the updating equation

$$
p_{j t \mid t}^{+}=\frac{\varphi\left(x_{t} \mid j_{t}=j, X_{t}, z_{t}, i_{t}, p_{t \mid t}\right)}{\varphi\left(x_{t} \mid X_{t}, z_{t}, i_{t}, p_{t \mid t}\right)} p_{j t \mid t} \quad\left(j \in N_{j}\right) .
$$

(ix) The policymaker and the private sector then leave period $t$ and enter period $t+1$, with the prior $p_{t+1 \mid t}$ given by the prediction equation

$$
p_{t+1 \mid t}=P^{\prime} p_{t \mid t}^{+}
$$


In the beginning of period $t+1$, the mode $j_{t+1}$ and the vector of shocks $\varepsilon_{t+1}$ are realized, and $X_{t+1}$ is determined by (1) and observed by the policymaker and private sector. The sequence of the nine steps above then repeats itself. For more detail on the explicit densities in the updating equations (21) and (25), see Svensson and Williams (2007b).

The transition equation for $p_{t+1 \mid t+1}$ can be written

$$
p_{t+1 \mid t+1}=Q\left(s_{t}, z_{t}, i_{t}, j_{t}, \varepsilon_{t}, j_{t+1}, \varepsilon_{t+1}\right),
$$

where $Q\left(s_{t}, z_{t}, i_{t}, j_{t}, \varepsilon_{t}, j_{t+1}, \varepsilon_{t+1}\right)$ is defined by the combination of (21) for period $t+1$ with (13) and (26). The equilibrium transition equation for the full state vector is then given by

$$
\begin{aligned}
s_{t+1} & \equiv\left[\begin{array}{c}
X_{t+1} \\
\Xi_{t} \\
p_{t+1 \mid t+1}
\end{array}\right]=\bar{g}\left(s_{t}, j_{t}, \varepsilon_{t}, j_{t+1}, \varepsilon_{t+1}\right) \\
& \equiv\left[\begin{array}{c}
A_{11 j_{t+1}} X_{t}+A_{12 j_{t+1}} x\left(s_{t}, j_{t}, \varepsilon_{t}\right) \\
+B_{1 j_{t+1}} i\left(s_{t}\right)+C_{1 j_{t+1}} \varepsilon_{t+1} \\
\gamma\left(s_{t}\right) \\
Q\left(s_{t}, z\left(s_{t}\right), i\left(s_{t}\right), j_{t}, \varepsilon_{t}, j_{t+1}, \varepsilon_{t+1}\right)
\end{array}\right],
\end{aligned}
$$

where the bottom block is given by the true updating equation (27) together with the policy function (16). Thus, we note that, in this AOP case, there is a distinction between the perceived transition and equilibrium transition equations, (15) and (18), which in the bottom block include the perceived updating equation, (7), and the true equilibrium transition equation, (28), which replaces the perceived updating equation, (7) with the true updating equation, (27).

Note that $V\left(s_{t}\right)$ in (19), which is subject to the perceived transition equation, (15), does not give the true (unconditional) value function for the AOP case. This is instead given by

$$
\bar{V}\left(s_{t}\right) \equiv \sum_{j} p_{j t t} \breve{V}\left(s_{t}, j\right)
$$

where the true conditional value function, $\breve{V}\left(s_{t}, j_{t}\right)$, satisfies

$$
\begin{aligned}
& \breve{V}\left(s_{t}, j\right)=\int\left\{\begin{array}{l}
L\left[X_{t}, x\left(s_{t}, j, \varepsilon_{t}\right), i\left(s_{t}\right), j\right] \\
+\delta \sum_{k} P_{j k} \breve{V}\left[\bar{g}\left(\begin{array}{l}
s_{t}, j, \varepsilon_{t}, \\
k, \varepsilon_{t+1}
\end{array}\right), k\right]
\end{array}\right\} \\
& \varphi\left(\varepsilon_{t}\right) \varphi\left(\varepsilon_{t+1}\right) d \varepsilon_{t} d \varepsilon_{t+1} \quad\left(j \in N_{j}\right) \text {. }
\end{aligned}
$$

That is, the true value function, $\bar{V}\left(s_{t}\right)$, takes into account the true updating equation for $p_{t \mid t},(27)$, whereas the optimal policy and the perceived value function, $V\left(s_{t}\right)$, in (19), are conditional on the perceived updating equation, (7), and thereby the perceived transition equation, (15). Note also that $\bar{V}\left(s_{t}\right)$ is the value function after $\tilde{X}_{t}$ has been observed but before $x_{t}$ is observed, so it is conditional on $p_{t \mid t}$ rather than $p_{t \mid t}^{+}$. Because the full transition equation, (28), is no longer linear due to the belief-updating equation, (27), the true value function, $\bar{V}\left(s_{t}\right)$, is no longer quadratic in $\tilde{X}_{t}$ for given $p_{t \mid t}$. Thus, more-complex numerical methods are required to evaluate losses in the AOP case, although policy is still determined simply as in the NL case.

As we discuss in Svensson and Williams (2007b), the difference between the true updating equation for $p_{t+1 \mid t+1},(27)$, and the perceived updating equation, (7), is that, in the true updating equation, $p_{t+1 \mid t+1}$ becomes a random variable from the point of view of period $t$, with mean equal to $p_{t+1 \mid t}$. This is because $p_{t+1 \mid t+1}$ depends on the realization of $j_{t+1}$ and $\varepsilon_{t+1}$. Thus Bayesian updating induces a mean-preserving spread over beliefs, which in turn sheds light on the gains from learning. If the conditional value function, $\breve{V}\left(s_{t}, j_{t}\right)$, under NL is concave in $p_{t \mid t}$ for given $\tilde{X}_{t}$ and $j_{t}$, then by Jensen's inequality the true expected future loss under AOP will be lower than the true expected future loss under NL. That is, the concavity of the value function for beliefs means that learning leads to lower losses. Although it is likely that $V$ is indeed concave, as we show in the applications, it need not be globally so and thus learning need not always reduce losses. In some cases, the losses incurred by increased variability of beliefs may offset the expected precision gains. Furthermore, under BOP, it may be possible to adjust policy to further increase the variance of $p_{t \mid t}$, that is, achieve a mean-preserving spread that might 
further reduce the expected future loss. ${ }^{12}$ This amounts to optimal experimentation.

\section{Bayesian Optimal Policy}

Finally, we consider the BOP case, when optimal policy is determined while taking the updating equation, (27), into account. That is, we now allow the policymaker to choose $i_{t}$ taking into account that his actions will affect $p_{t+1 \mid t+1}$, which in turn will affect future expected losses. In particular, experimentation is allowed and is optimally chosen. For the BOP case, there is hence no distinction between the perceived and true transition equation.

The transition equation for the BOP case is

$$
\begin{aligned}
s_{t+1} & \equiv\left[\begin{array}{c}
X_{t+1} \\
\Xi_{t} \\
p_{t+1 \mid t+1}
\end{array}\right]=g\left(s_{t}, z_{t}, i_{t}, \gamma_{t}, j_{t}, \varepsilon_{t}, j_{t+1}, \varepsilon_{t+1}\right) \\
& \equiv\left[\begin{array}{c}
A_{11 j_{t+1}} X_{t}+A_{12 j_{t+1}} \tilde{x}\left(s_{t}, z_{t}, i_{t}, j_{t}, \varepsilon_{t}\right) \\
+B_{1 j_{t+1}} i_{t}+C_{1 j_{t+1}} \varepsilon_{t+1} \\
\gamma_{t} \\
Q\left(s_{t}, z_{t}, i_{t}, j_{t}, \varepsilon_{t}, j_{t+1}, \varepsilon_{t+1}\right)
\end{array}\right] .
\end{aligned}
$$

Then the dual optimization problem can be written as (12) subject to the above transition equation (30). However, in the Bayesian case, matters simplify somewhat, as we do not need to compute the conditional value functions, $\hat{V}\left(s_{t}, j_{t}\right)$, which we recall were required because of the failure of the law of iterated expectations in the AOP case. We note now that the second term on the right side of (12) can be written as

$$
\mathrm{E}_{t} \hat{V}\left(s_{t+1}, j_{t+1}\right) \equiv \mathrm{E}\left[\hat{V}\left(s_{t+1}, j_{t+1}\right) \mid s_{t}\right] .
$$

Because, in the Bayesian case, the beliefs do satisfy the law of iterated expectations, this is then the same as

$$
\mathrm{E}\left[\hat{V}\left(s_{t+1}, j_{t+1}\right) \mid s_{t}\right]=\mathrm{E}\left[\tilde{V}\left(s_{t+1}\right) \mid s_{t}\right] .
$$

\footnotetext{
${ }^{12}$ Kiefer (1989) examines the properties of a value function, including concavity, under Bayesian learning for a simpler model without forward-looking variables.
}

See Svensson and Williams (2007b) for a proof.

Thus, the dual Bellman equation for the Bayesian optimal policy is

$$
\begin{aligned}
& \tilde{V}\left(s_{t}\right)\left.=\max _{\gamma_{t}} \min _{\left(z_{t}, i_{t}\right)} \mathrm{E}_{t}\left\{\begin{array}{l}
\tilde{L}\left(\tilde{X}_{t}, z_{t}, i_{t}, \gamma_{t}, j_{t}, \varepsilon_{t}\right) \\
+\delta \tilde{V}\left[g\left(s_{t}, z_{t}, i_{t}, \gamma_{t}, j_{t}, \varepsilon_{t}, j_{t+1}, \varepsilon_{t+1}\right)\right.
\end{array}\right]\right) \\
& \equiv \max _{\gamma_{t}} \min _{\left(z_{t}, i_{t}\right)} \sum_{j} p_{j t t t} \int\left[\begin{array}{l}
\tilde{L}\left(\tilde{X}_{t}, z_{t}, i_{t}, \gamma_{t}, j, \varepsilon_{t}\right) \\
+\delta \sum_{k} P_{j k} \tilde{V}\left[g\left(\begin{array}{l}
s_{t}, z_{t}, i_{t}, \gamma_{t}, \\
j, \varepsilon_{t}, k, \varepsilon_{t+1}
\end{array}\right)\right]
\end{array}\right] \\
& \varphi\left(\varepsilon_{t}\right) \varphi\left(\varepsilon_{t+1}\right) d \varepsilon_{t} d \varepsilon_{t+1},
\end{aligned}
$$

where the transition equation is given by (30).

The solution to the optimization problem can be written

$$
\begin{aligned}
\tilde{I}_{t} & \equiv\left[\begin{array}{c}
z_{t} \\
i_{t} \\
\gamma_{t}
\end{array}\right]=\tilde{I}\left(s_{t}\right) \equiv\left[\begin{array}{c}
z\left(s_{t}\right) \\
i\left(s_{t}\right) \\
\gamma\left(s_{t}\right)
\end{array}\right] \\
& =F\left(\tilde{X}_{t}, p_{t \mid t}\right) \equiv\left[\begin{array}{c}
F_{z}\left(\tilde{X}_{t}, p_{t \mid t}\right) \\
F_{i}\left(\tilde{X}_{t}, p_{t \mid t}\right) \\
F_{\gamma}\left(\tilde{X}_{t}, p_{t \mid t}\right)
\end{array}\right], \\
X_{t} & =x\left(s_{t}, j_{t}, \varepsilon_{t}\right) \equiv \tilde{X}\left(X_{t}, z\left(s_{t}\right), i\left(s_{t}\right), j_{t}, \varepsilon_{t}\right) \\
& \equiv F_{x}\left(\tilde{X}_{t}, p_{t \mid t}, j_{t}, \varepsilon_{t}\right) .
\end{aligned}
$$

Because of the nonlinearity of (27) and (30), the solution is no longer linear in $\tilde{X}_{t}$ for given $p_{t \mid t}$. The dual value function, $\tilde{V}\left(s_{t}\right)$, is no longer quadratic in $\tilde{X}_{t}$ for given $p_{t \mid t}$. The value function of the primal problem, $V\left(s_{t}\right)$, is given by, equivalently, (19), (29) (with the equilibrium transition equation (28) with the solution (32)), or

$$
\begin{aligned}
& V\left(s_{t}\right)=\sum_{j} p_{j t t t}\left\{\begin{array}{l}
L\left[X_{t}, x\left(s_{t}, j, \varepsilon_{t}\right), i\left(s_{t}\right), j\right] \\
+\delta \sum_{k} P_{j k} V\left[\bar{g}\left(s_{t}, j, \varepsilon_{t}, k, \varepsilon_{t+1}\right)\right]
\end{array}\right\} \\
& \varphi\left(\varepsilon_{t}\right) \varphi\left(\varepsilon_{t+1}\right) d \varepsilon_{t} d \varepsilon_{t+1} .
\end{aligned}
$$

It is also no longer quadratic in $\tilde{X}_{t}$ for given $p_{t \mid t}$. Thus, more complex and detailed numerical methods are necessary in this case to find the optimal policy and the value function. Therefore, little can be said in general about the solution of 
the problem. Nonetheless, in numerical analysis it is very useful to have a good starting guess at a solution, which in our case comes from the AOP case. In our examples below we explain in more detail how the BOP and AOP cases differ and what drives the differences.

\section{Observable Modes}

In this paper we largely focus on the cases where the policymakers do not observe the current mode, which is certainly the more relevant case when analyzing model uncertainty. However, some situations may arguably be better modeled by observable shifts in modes, as in most of the econometric literature on regime-switching models. Moreover, one way to gauge the effects of uncertainty in a model is to move from a constantcoefficient specification to one in which the parameters are observable but may vary. (That is, the current values of parameters are known, but future values are uncertain.) For this reason, we use the observable mode case, to analyze implications of uncertainty on policy. In Svensson and Williams (2007a), we develop simple algorithms for observable changes in modes, which play off the fact that conditional on the mode the evolution of the economy is linear and preferences are quadratic. Thus, the optimal policy consists of a mode-dependent collection of linear policy rules and can be written

$$
i_{t}=F_{i j_{t}} \tilde{X}_{t}
$$

for $j_{t} \in N_{j}$.

\section{LEARNING AND \\ EXPERIMENTATION IN A SIMPLE NEW KEYNESIAN MODEL}

\section{The Model}

For our policy exercises, we consider a benchmark hybrid New Keynesian Phillips curve (see Woodford, 2003, for an exposition):

$$
\pi_{t}=\left(1-\omega_{j_{t}}\right) \pi_{t-1}+\omega_{j_{t}} \mathrm{E}_{t} \pi_{t+1}+\gamma_{j_{t}} y_{t}+c \varepsilon_{t}
$$

Here $\pi_{t}$ is the inflation rate, $y_{t}$ is the output gap, $\omega_{j_{t}}$ is a parameter reflecting the degree of forwardlooking behavior in price setting, and $\gamma_{j_{t}}$ is a composite parameter reflecting the elasticity of demand and frequency of price adjustment. For simplicity, we assume that policymakers can directly control the output gap, $y_{t}$. In another paper, Svensson and Williams (2008), we consider optimal policy in the standard two-equation New Keynesian model that also includes a loglinearized consumption Euler equation. Many of the same issues that we focus on here arise there as well, but the simpler setting in the present paper allows us to focus more directly on the effects of uncertainty on policy.

We focus on two key sources of uncertainty in the New Keynesian Phillips curve. Our first example considers the degree of forward-looking behavior in inflation. In the model, this translates to uncertainty about $\omega_{j}$. If this parameter is large, inflation is largely determined by current shocks and expectations of the future, whereas if $\omega_{j}$ is small, then there is a substantial exogenous inertia in the inflation process. Our second example analyzes uncertainty about the slope of the Phillips curve, as reflected in the parameter $\gamma_{j}$. This could reflect changes in the degree of monopolistic competition (which also lead to varying markups) and/or changes in the degree of price stickiness. In each example, we look first at the effect of uncertainty, going from a constant-coefficient model to a model with random coefficients. Then, we analyze the effects of learning and experimentation on policy and losses.

In both examples, we use the following loss function:

$$
L_{t}=\pi_{t}^{2}+\lambda y_{t}^{2}
$$

We set the loss-function parameters as $\delta=0.98$, $\lambda=0.1$, and set the shock standard deviation to $c=0.5$. Even though different structural parameters vary in the two examples, both examples use two modes and set the transition matrix to

$$
P=\left[\begin{array}{ll}
0.98 & 0.02 \\
0.02 & 0.98
\end{array}\right] .
$$




\section{Figure 1}

\section{Policies and Losses from Observable and Constant Modes}
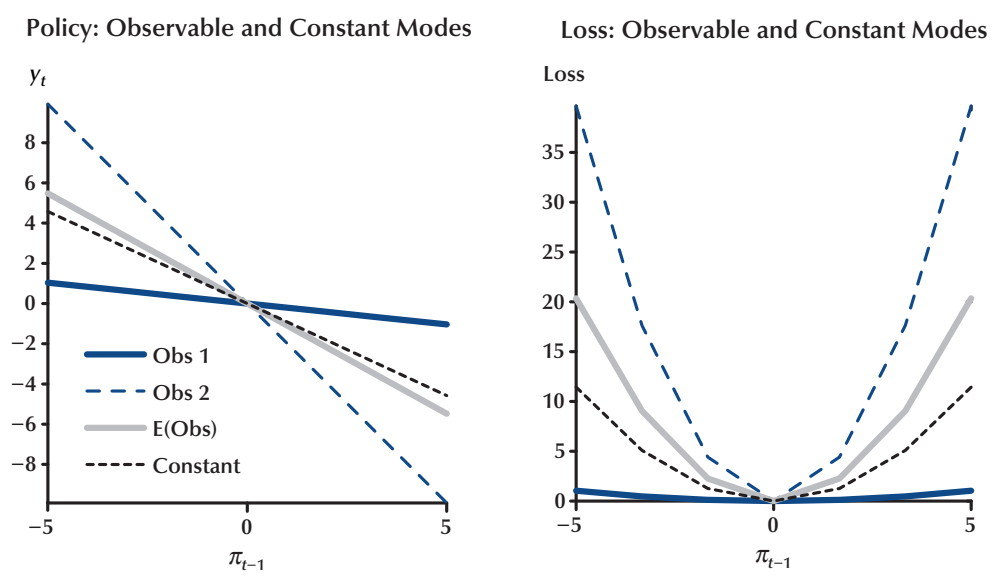

NOTE: Obs 1 (2) is observable mode 1 (2); $E(O b s)$ is the unconditional average policy.

In both examples, we examine the value functions and optimal policies for this simple New Keynesian model under NL, AOP, and BOP. We have one forward-looking variable $\left(x_{t} \equiv \pi_{t}\right)$ and consequently one Lagrange multiplier $\left(\Xi_{t-1} \equiv\right.$ $\left.\Xi_{\pi, t-1}\right)$. We have one predetermined variable $\left(X_{t} \equiv \pi_{t-1}\right)$ and the estimated mode probabilities $\left(p_{t \mid t} \equiv\left(p_{1 t \mid t}, p_{2 t \mid t}\right)^{\prime}\right.$, of which we only need keep track of one, $\left.p_{1 t \mid t}\right)$. Thus, the value and policy functions, $V\left(s_{t}\right)$ and $i\left(s_{t}\right)$, are all three dimensional $\left(s_{t}=\left(\pi_{t-1}, \Xi_{\pi, t-1}, p_{1 \mid t}\right)^{\prime}\right)$. For computational reasons, we are forced to restrict attention to relatively sparse grids with few points. The following plots show two-dimensional slices of the value and policy functions, focusing on the dependence on $\pi_{t-1}$ and $p_{1 t \mid t}$ (which we for simplicity denote by $p_{1 \mid t}$ in the figures). In particular, all of the plots are for $\Xi_{\pi, t-1}=0$.

\section{Example 1: How Forward-Looking Is Inflation?}

This example analyzes one of the main sources of uncertainty in the New Keynesian frameworkthe degree to which inflation is a forward-looking variable responding to expectations of future developments. Specifications that suggest that inflation has substantial exogenous persistence have tended to fit better empirically, while perhaps being less rigorous in their micro-foundations. In this example, we see how uncertainty about the degree of forward-looking behavior, as indexed by $\omega_{j}$, affects policy. Thus, we assume that there are two modes, one more forward looking, with $\omega_{1}=0.8$, and the other more backward looking, with $\omega_{2}=0.2$. Note that, with the transition matrix $P$ as specified above, this means $E\left(\omega_{j}\right)=0.5$. For this example, we fix the slope parameter at $\gamma=0.1$.

In Figure 1, we illustrate the effects of uncertainty on policy and losses. In the left panel, we plot the two mode-dependent optimal policy functions for the MJLQ model with observable modes, labeled "Obs 1 " for mode 1 and "Obs 2" for mode 2. Here, we see that the optimal policy is more aggressive in the more backward-looking mode 2, because in response to a higher inflation the optimal policy involves larger negative output gaps. The unconditional average policy is labeled "E(Obs)" and shown with a gray line. For comparison, the constant-coefficient case, where we set $\omega_{1}=\omega_{2}=E\left(\omega_{j}\right)=0.5$, is plotted with a black dashed line. Here, we see that optimal policy under uncertainty is more aggressive in respond- 


\section{Figure 2}

\section{Losses and Differences in Losses from NL, AOP, and BOP}
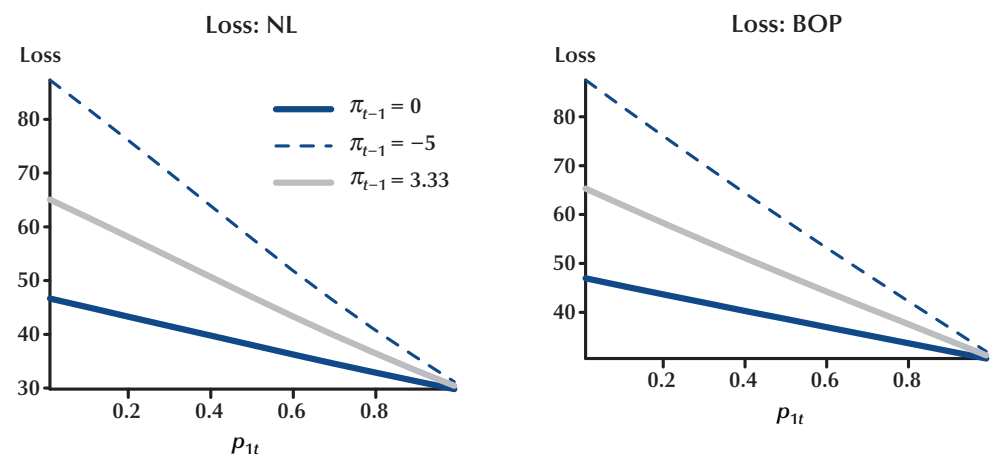

Loss Differences: AOP-NL

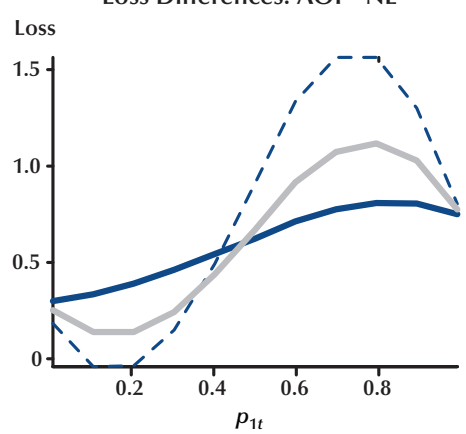

Loss Differences: BOP-AOP

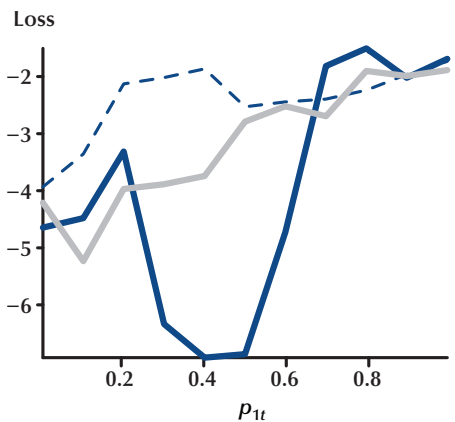

ing to inflation movements than optimal policy in the absence of uncertainty.

A common starting point for thinking about the effects of uncertainty on policy is Brainard's (1967) classic analysis, which suggested that uncertainty should make policy more cautious. However, Brainard worked in a static framework and the source of uncertainty he analyzed was a slope coefficient on how policy affects the economy. Our second example below is closer to Brainard's and comes to similar conclusions. But, in this example, our results suggest, at least for this parameterization, that uncertainty about the dynamics of inflation leads to more-aggressive policy. This is similar to what Söderström (2002) found in a backward-looking model.

The right panel of Figure 1 plots the losses associated with the optimal policies in the different cases. When inflation is more forward looking, it is easier to control and so overall losses are lower even with less-aggressive policies. However, uncertainty about the dynamics of inflation can have significant effects on losses for moderate to high inflation levels. This is evident by comparing the constant-coefficient and average observable curves, where we see that the loss nearly doubles at the edges of the plot.

Now we keep the same specification, but make the more realistic assumption that the current mode is not observed. Thus, we analyze the effects of learning and experimentation on policy and losses. The top-two panels of Figure 2 show losses under NL and BOP as functions of $p_{1 t}$. The bottomtwo panels of the figure show the differences between losses under NL, AOP, and BOP. Figure 3 shows the corresponding policy functions and their differences. The top-two panels plot the policy functions under AOP and BOP as a function of inflation. The AOP policy is linear in $\pi_{t}$, and clearly the BOP policy is nearly so. The bot- 
Figure 3

\section{Optimal Policies and Their Differences Under AOP and BOP}
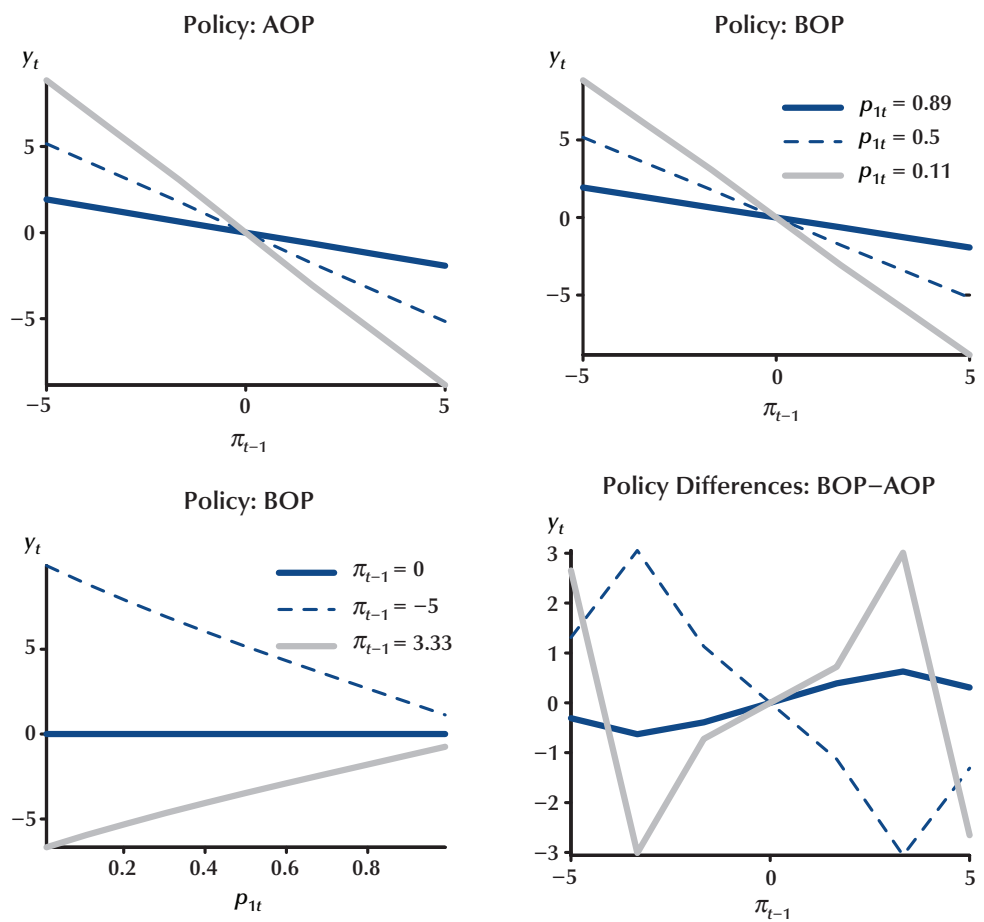

tom-left panel plots the BOP policy as a function of $p_{1 t}$, showing that policy is less aggressive (that is, has a smaller magnitude of response) the greater is the probability of being in the more forwardlooking mode 1 . The bottom-right panel shows that the policy differences between AOP and BOP, the experimentation component of policy, are incredibly small.

In Svensson and Williams (2007b), we show that learning implies a mean-preserving spread of the random variable $p_{t+1 \mid t+1}$ (which under learning is a random variable from the vantage point of period $t$ ). Hence, concavity of the value function under NL in $p_{1 t}$ implies that learning is beneficial, because then a mean-preserving spread reduces the expected future loss. However, we see in Figure 2 that the value function is actually slightly convex in $p_{1 t}$, so learning is not beneficial here. Consequently, we see in Figure 2 that AOP gives higher losses than NL. In contrast, for a backward-looking example in Svensson and
Williams (2007b), the value function is concave and learning is beneficial. Experimentation is beneficial here, as BOP does give lower losses than AOP, but the difference is minuscule. So, for this example, learning has sizable effects on losses and is detrimental, whereas experimentation is beneficial but has negligible effects.

Why would learning not be beneficial with forward-looking variables? It may at least partially be a remnant of our assumption of symmetric beliefs and information between the private sector and the policymaker. With backward-looking models, we have generally found that learning is beneficial. However, under our assumption of symmetric information and beliefs between the private sector and the policymaker, both the private sector and the policymaker learn. The difference between backward- and forward-looking models then comes from the way that private sector beliefs also respond to learning. Having more reactive private sector beliefs may add volatility 


\section{Figure 4}

\section{Policies and Losses from Observable and Constant Modes}
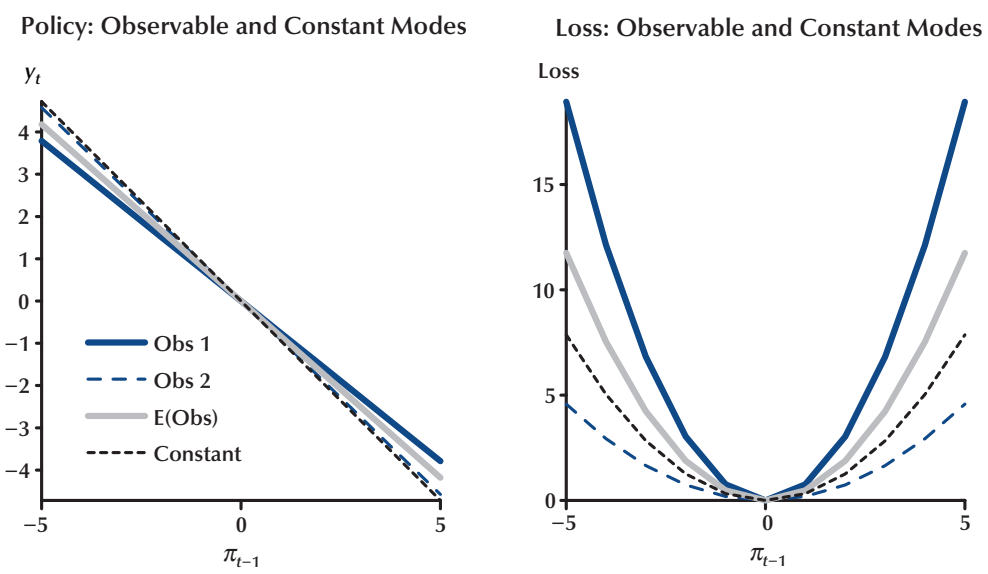

NOTE: Obs 1 (2) is observable mode 1 (2); $E(O b s)$ is the unconditional average policy.

and make it more difficult for the policymaker to stabilize the economy.

\section{Example 2: What Is the Slope of the Phillips Curve?}

This example analyzes the other main source of uncertainty in the New Keynesian Phillips curve-the extent to which inflation responds to fluctuations in the output gap. Once again, we assume that there are two modes: Now one has a Phillips curve that is flatter, with $\gamma_{1}=0.05$, and the other has a steeper curve, with $\gamma_{2}=0.25$. Note that with the transition matrix $P$ as specified above, this means $E\left(\gamma_{j}\right)=0.15$. For this example, we fix the forward-looking expectations parameter at $\omega=0.5$. Because policymakers once again directly control the output gap, this example is a forward-looking counterpart to the classic Brainard (1967) analysis of uncertainty about the effectiveness of the control.

In Figure 4 we illustrate the effects of uncertainty on policy and losses. As in the previous example, the left panel plots the two modedependent optimal policy functions for the MJLQ model with observable modes. Here, we see that the MJLQ optimal policies in both modes are less aggressive than the constant-coefficient case. Thus, our results here are in accord with Brainard'suncertainty about the slope of the Phillips curve leads to more cautious policy.

The right panel of Figure 4 plots the losses associated with the optimal policies in the different cases. When the Phillips curve is steeper, inflation responds more to the output gap, making inflation easier to control. Thus, overall losses are lower in mode 2, even with less-aggressive policies. However, once again uncertainty about this key parameter can have significant effects on losses for high inflation levels. This is evident by comparing the constant-coefficient and average observable curves, where we see that the loss nearly doubles at the edges of the plot.

Now we again keep the same specification, but make the more realistic assumption that the current mode is not observed. The top-two panels of Figure 5 show losses under NL and BOP as functions of $p_{1 t}$. The bottom-two panels of the figure show the differences between losses under NL, AOP, and BOP. We see in Figure 2 that the value function is once again slightly convex in $p_{1 t}$, so learning is not beneficial here. Consequently, we see in the bottom-right panel of Figure 2 that AOP gives higher losses than NL. Thus, once 
Figure 5

\section{Losses and Differences in Losses from NL, AOP, and BOP}
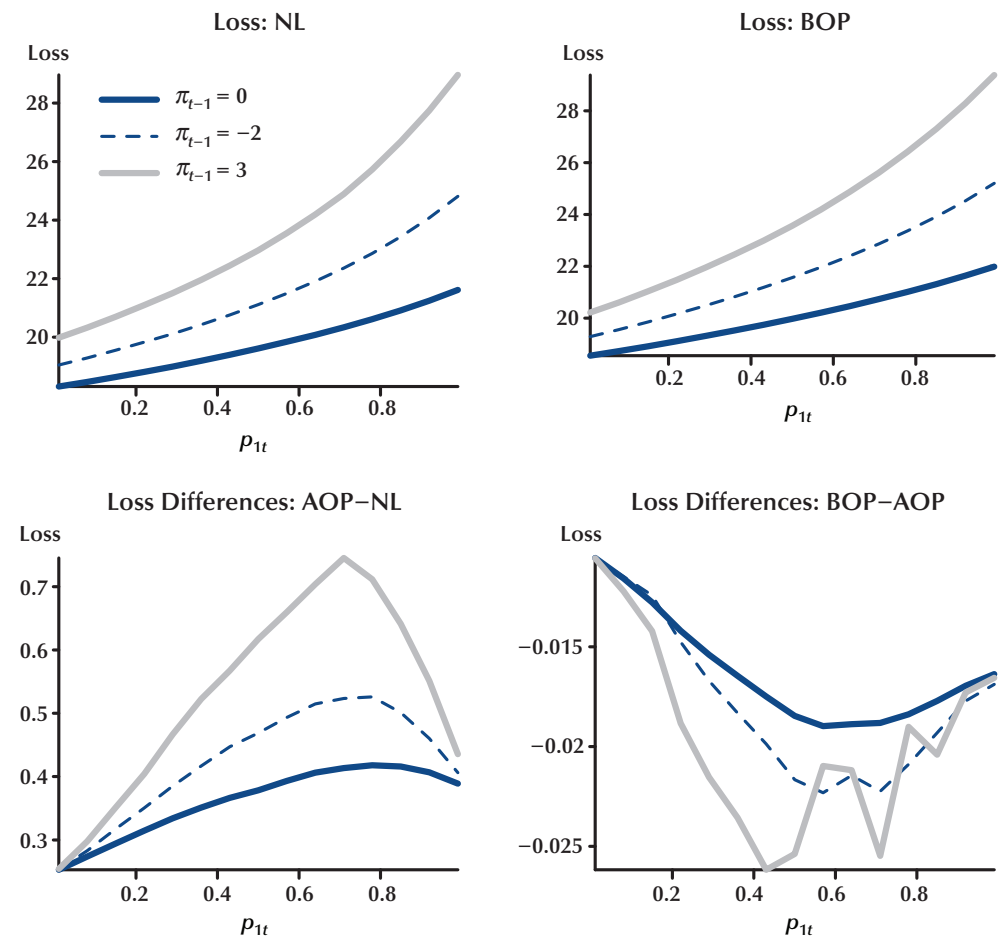

again, the additional volatility outweighs the improved inference and makes learning detrimental in this example. Experimentation is once again beneficial, as BOP gives lower losses than AOP. And, while the effects of experimentation are an order of magnitude smaller than the effects of learning, the gains from recognizing the endogeneity of information are nonnegligible here. Thus, for uncertainty about the slope of the Phillips curve, policymakers may have an incentive to experiment-that is, to take actions to mitigate future uncertainty.

Figure 6 shows the corresponding policy functions and their differences. The top-two panels plot the policy functions under AOP and BOP as a function of inflation. The AOP policy is linear in $\pi_{t-1}$, and clearly the BOP policy is nearly so, although some differences are evident at the edge of the plot. The bottom-left panel plots the BOP policy as a function of $p_{1 t}$, showing that the policy function is relatively flat in this dimension. The bottom-right panel plots the difference between the AOP and BOP policy functions, which shows that here the experimentation motive leads toward less-aggressive policy. This is counter to an example in Svensson and Williams (2007b), where we show that in a backward-looking model experimentation may lead to more-aggressive policy. There, policy makes outcomes more dispersed in order to sharpen inference over the modes. However, here, because learning is detrimental, the experimentation component of policy seeks to slow the effects of learning by making outcomes less dispersed. This serves to illustrate that the experimentation component of policy need not be associated with wild or aggressive policy action, but rather it optimally takes into account how information influences the targets of policy. 


\section{Figure 6}

\section{Optimal Policies and Their Differences Under AOP and BOP}
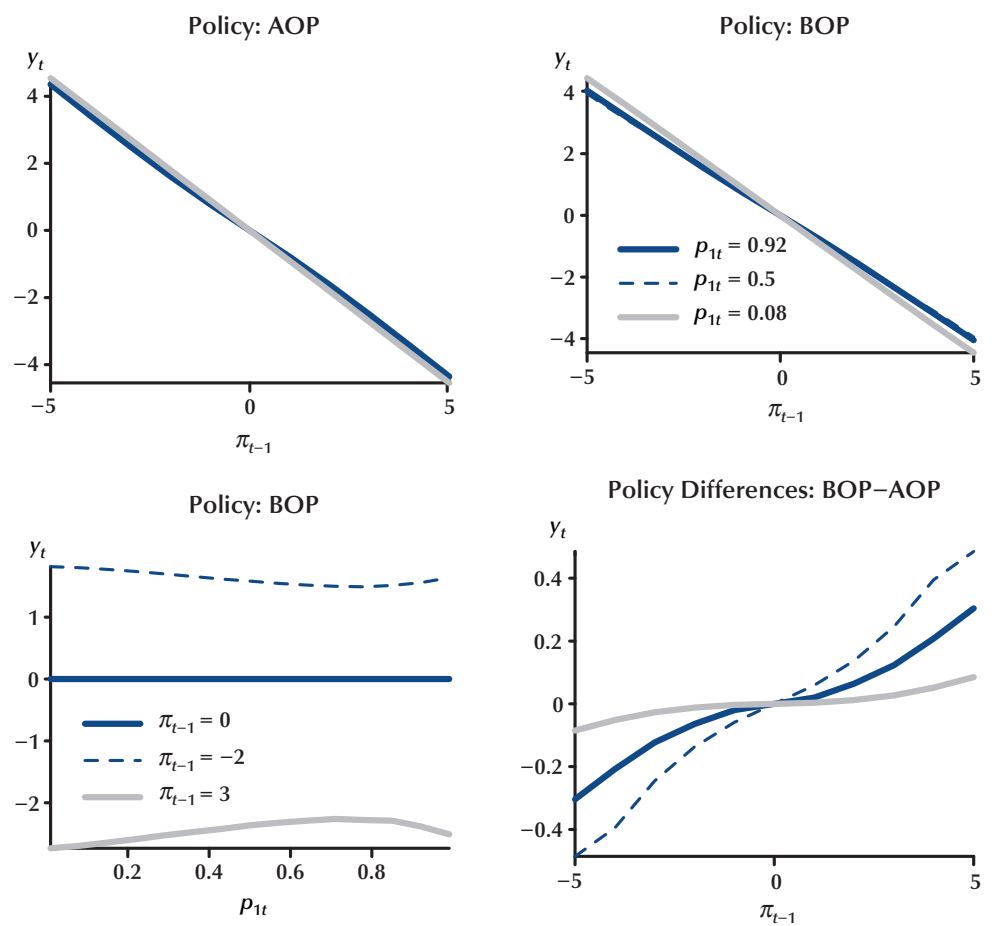

\section{CONCLUSION}

In this paper, we have presented a relatively general framework for analyzing model uncertainty and the interactions between learning and optimization. Although this is a classic issue, very little to date has been done for systems with forward-looking variables, which are essential elements of modern models for policy analysis. Our specification is general enough to cover many practical cases of interest, yet remains relatively tractable in implementation. This is definitely true for cases when decisionmakers do not learn from the data they observe (our case of no learning, $\mathrm{NL}$ ) or when they do learn but do not account for learning in optimization (our case of adaptive optimal policy, AOP). In both of these cases, we have developed efficient algorithms for solving for the optimal policy, which can handle relatively large models with multiple modes and many state variables. However, in the case of the
Bayesian optimal policy (BOP), where the experimentation motive is taken into account, we must solve more-complex numerical dynamic programming problems. Thus, to fully examine optimal experimentation, we are haunted by the curse of dimensionality, forcing us to study relatively small and simple models.

Thus, an issue of much practical importance is the size of the experimentation component of policy and the losses entailed by abstracting from it. Although our results in this paper are far from comprehensive, they suggest that in practical settings the experimentation motive may not be a concern. The above and similar examples that we have considered indicate that the benefits of learning (moving from NL to AOP) may be substantial, whereas the benefits from experimentation (moving from AOP to BOP) are modest or even insignificant. If this preliminary finding stands up to scrutiny, experimentation in economic policy in general and monetary policy in 


\section{Svensson and Williams}

particular may not be very beneficial, in which case there is little need to face the difficult ethical and other issues involved in conscious experimentation in economic policy. Furthermore, the AOP is much easier to compute and implement than the BOP. To have this truly be a robust implication, more simulations and cases need to be examined.

\section{REFERENCES}

Beck, Günter W. and Wieland, Volker. "Learning and Control in a Changing Economic Environment." Journal of Economic Dynamics and Control, August 2002, 25(9-10), pp. 1359-77.

Blake, Andrew P. and Zampolli, Fabrizio. "Time Consistent Policy in Markov Switching Models with Rational Expectations.” Working Paper No. 298, Bank of England, 2006.

Brainard, William C. "Uncertainty and the Effectiveness of Policy." American Economic Review, May 1967, 57(2), pp. 411-25.

Cogley, Timothy; Colacito, Riccardo and Sargent, Thomas J. "The Benefits from U.S. Monetary Policy Experimentation in the Days of Samuelson and Solow and Lucas." Journal of Money, Credit, and Banking, February 2007, 39(2), pp. 67-99.

Costa, Oswaldo L.V.; Fragoso, Marecelo D. and Marques, Ricardo P. Discrete-Time Markov Jump Linear Systems. London: Springer, 2005.

do Val, João B.R. and Başar, Tamer. "Receding Horizon Control of Jump Linear Systems and a Macroeconomic Policy Problem.” Journal of Economic Dynamics and Control, August 1999, 23(8), pp. 1099-31.

Ellison, Martin. "The Learning Cost of Interest Rate Reversals." Journal of Monetary Economics, November 2006, 53(8), pp. 1895-907.

Ellison, Martin and Valla, Natacha. "Learning, Uncertainty and Central Bank Activism in an Economy with Strategic Interactions." Journal of Monetary Economics, August 2001, 48(1), pp. 153-71.
Evans, George and Honkapohja, Seppo. Learning and Expectations in Macroeconomics. Princeton, NJ: Princeton University Press, 2001.

Kiefer, Nicholas M. "A Value Function Arising in the Economics of Information." Journal of Economic Dynamics and Control, April 1989, 13(2), pp. 201-23.

Lindé, Jesper. "Estimating New-Keynesian Phillips Curves: A Full Information Maximum Likelihood Approach." Journal of Monetary Economics, September 2005, 52(6), pp. 1135-49.

Marcet, Albert and Marimon, Ramon. "Recursive Contracts." Working paper, Universitat Pompeu Fabra, Department of Economics and Business, 1998; www.econ.upf.edu.

Poole, William. "A Policymaker Confronts Uncertainty." Federal Reserve Bank of St. Louis Review, September 1998, 80(5), pp. 3-8.

Rudebusch, Glenn D. and Svensson, Lars E.O. "Policy Rules for Inflation Targeting," in John B. Taylor, ed., Monetary Policy Rules. Chicago: University of Chicago Press, 1999.

Söderström, Ulf. "Monetary Policy with Uncertain Parameters." Scandinavian Journal of Economics, 2002, 104(1), pp. 125-45.

Svensson, Lars E.O. “Optimization under Commitment and Discretion, the Recursive Saddlepoint Method, and Targeting Rules and Instrument Rules.” Lecture notes, Princeton University, 2007;

www.princeton.edu/svensson.

Svensson, Lars E.O. and Williams, Noah. "Monetary Policy with Model Uncertainty: Distribution Forecast Targeting." Working paper, Princeton University, May 2007a; www.princeton.edu/svensson/.

Svensson, Lars E.O. and Williams, Noah. "Bayesian and Adaptive Optimal Policy Under Model Uncertainty.” NBER Working Paper No. 13414, National Bureau of Economic Research, 2007b.

Svensson, Lars E.O. and Williams, Noah. "Optimal Monetary Policy in DSGE Models: A Markov JumpLinear-Quadratic Approach.” NBER Working Paper 
No. W13892, National Bureau of Economic Research, 2008.

Tesfaselassie, Mewael F.; Schaling, Eric and Eijffinger, Sylvester C.W. "Learning about the Term Structure and Optimal Rules for Inflation Targeting." CEPR Discussion Paper No. 5896, Centre for Economic Policy Research, 2006.

Wieland, Volker. "Learning by Doing and the Value of Optimal Experimentation." Journal of Economic Dynamics and Control, March 2000, 24(4),

pp. 501-34.

Wieland, Volker. "Monetary Policy and Uncertainty about the Natural Unemployment Rate: BrainardStyle Conservatism versus Experimental Activism." Advances in Macroeconomics, March 2006, 6(1), pp. 1-34.

Woodford, Michael. Interest and Prices: Foundations of a Theory of Monetary Policy. Princeton, NJ: Princeton University Press, 2003.

Zampolli, Fabrizio. "Optimal Monetary Policy in a Regime-Switching Economy: The Response to Abrupt Shifts in Exchange-Rate Dynamics." Working Paper No. 297, Bank of England, 2006. 
